\title{
Long range correlations, event simulation and parton percolation
}

\author{
C. Pajares \\ IGFAE and Departamento de Física de Partículas, Univ. of Santiago de Compostela, 15706, Santiago \\ de Compostela, Spain
}

\begin{abstract}
We study the RHIC data on long range rapidity correlations, comparing their main trends with different string model simulations. Particular attention is paid to color percolation model and its similarities with color glass condensate. As both approaches corresponds, at high density, to a similar physical picture, both of them give rise to a similar behavior on the energy and the centrality of the main observables. Color percolation explains the transition from low density to high density.
\end{abstract}

Keywords: Long range correlations, color percolation, color glass condensate

\section{Introduction}

Forward- backward multiplicity correlations have been studied recently in RHIC experiments [1] [2] [3] STAR data [1] has measured the parameter b, defined by

$$
b=\frac{<n_{F} n_{B}>-<n_{F}><m_{B}>}{<n_{F}^{2}>-<n_{F}>^{2}}=\frac{D_{B F}^{2}}{D_{F F}^{2}}
$$

where $D_{B F}$ and $D_{F F}$ are the backward-forward and forward-forward dispersions respectively. In between the forward and backward rapidities bins there is a rapidity gap $\Delta \eta$, being measured b as a function of $\Delta \eta$. FB multiplicity correlations probe the longitudinal characteristics of the system produced in AA and hh collisions. The presence of sizable long range rapidity correlations was predicted in some particle models, as the Dual Parton Model (DPM) [4] [5] and the Color Glass Condensate (CGC) model [6] [7] [8] that contain longitudinal color flux tubes in CGC. The STAR data at $200 \mathrm{GeV}$ show that from most central $(0-10 \%)$ to mid- peripheral $(40-50 \%) \mathrm{Au}-\mathrm{Au}$ collisions there is a general decrease in the FB correlation strength $\mathrm{b}$. The shape of $\mathrm{b}$ for $(40-50 \%)$ centrality as a function of $\Delta \eta$ indicates a fast decreasing with increasing $\Delta \eta$. If only short range correlations contributed to $\mathrm{b}$, all centralities should resemble the $(40-50 \%)$ result, however b remains flat in the whole range of $\Delta \eta$ even for $\Delta \eta>1.5$ for $20-30 \%$, $10-20 \%, 0-10 \%$ centralities, indicating long range correlation. In pp the $\mathrm{b}$ value is much smaller, decreasing with increasing $\Delta \eta$, being close to zero for $\Delta \eta>1.2$.

Not all the strings models can reproduce data. First of all the strings must be extended in both hemispheres. There are models like Hijing, that the strings are formed between the partons of the same nucleon, there is not color exchange between projectile Preprint submitted to Elsevier

November 8, 2018 
and target, and the fragmentation of these strings gives rise to particles that most of them are produced only in one rapidity hemisphere and therefore it is not able to reproduce the b shape. The inclusion of rescattering between partons of different nucleons, as it is done in PACIAE model (based on HIJING) improves the comparison with the experimental data and it can reproduce the strength and shape of $b$ for central but not for peripherical collisions 9 ].

In modified wounded nucleon of Bzdak [10] is explained the enhancement of b with respect to the elementary pp interaction by the asymmetric shape of the rapidity density of produced particles from a single wounded nucleon in addition to the fluctuations in the number of wounded nucleons in the colliding nuclei. This asymmetric shape means that the particles are not produced only in one hemisphere. However, in this model the length of the correlations is the same for $\mathrm{pp}$ than for AA central collisions, contrary to the experimental data. The string percolation [11] [12] and the CGC models [13] [14] have the right longitudinal structure able to explain at least qualitatively, the dependence on the energy and centrality of the strength and length of the long range rapidity correlations. Both of them predict a very long rapidity correlations for AA collisions at LHC energies, indeed more than eight units of rapidity. As far as the length of the long range correlations establishes the length of the near-side ridge structure, it is predicted a very long ridge at LHC.

\section{The string percolation model}

Multiparticle production is currently described in terms of color strings stretched between the partons of the projectile and the target. These strings decay into new ones, via $q-\bar{q}$ production, (Schwinger mechanism), and subsequently hadronize to produce observed hadrons. Color strings may be viewed as small areas in the transverse space, $S_{1}=\pi r_{0}^{2}$, with $r_{0} \simeq .2-.3 \mathrm{fm}$, filled with the color field created by the colliding partons. With increasing energy and/or atomic number of colliding particles, the number of exchange string grows, and they start to overlap, forming clusters, very much like disk in 2- dimensional percolation. Each cluster has a higher color field and an energymomentum that correspond to the sum of the energy momentum of the overlapping strings. At a certain critical density $\eta_{c} \simeq 1.2-1.5$ a macroscopical cluster appears which marks the percolation phase transition. The variable $\eta$ used above is defined by $\eta_{c}=N_{s} \frac{S_{1}}{S_{A}}$, where $N_{s}$ is the number of strings, which is proportional to the number of collisions $N_{s} \sim N_{A}^{\frac{4}{3}}$, and $S_{A}$ is the overlapping area. At $b=0, S_{A}=\pi R_{A}^{2}$.

The color field $\vec{Q}_{n}$ inside the cluster is the vectorial sum of the color field $\overrightarrow{Q_{1}}$ of each individual string. The resulting color field covers the area $S_{n}$ of the cluster. As $\overrightarrow{Q_{n}^{2}}=\left(\sum \overrightarrow{Q_{1}}\right)^{2}$, and the individual string colors may be oriented in an arbitrary manner respective to one another, the average $\overrightarrow{Q_{1 i}} \overrightarrow{Q_{1 j}}$ is zero and $\overrightarrow{Q_{n}^{2}}=n \overrightarrow{Q_{1}} \overrightarrow{Q_{1}}$ depends also on the area $S_{1}$ as well on the total area of the cluster $S_{n}$, therefore

$$
Q_{n}=\sqrt{\frac{n S_{n}}{S_{1}}} Q_{1}
$$

in such a way that if the strings are just touching each other $S_{n}=n S_{1}$ and $Q_{n}=n S_{1}$, so the strings are independent of each other. On the contrary if they are fully overlap, 
$S_{n}=S_{1}$ and $Q_{n}=\sqrt{n} Q_{1}$. from (2) we can deduce that the mean multiplicity $\mu_{n}$ and the mean $\left\langle p_{T}^{2}>_{n}\right.$ of the particles produced by the cluster of $\mathrm{n}$ strings.

$$
\mu_{n}=\sqrt{\frac{n S_{n}}{S_{1}}} \mu_{1}, \quad<p_{T}^{2}>_{n}=\sqrt{\frac{n S_{1}}{S_{n}}}<p_{T}^{2}>_{1}
$$

As we have obtained [12].

$$
<n \frac{S_{1}}{S_{n}}>=\frac{\eta}{1-e^{-\eta}}=\frac{1}{F(\eta)^{2}}
$$

where $1-e^{-\eta}$ is the fraction of the total area covered by the strings, we can write for the multiplicity and $<p_{T}^{2}>$

$$
\mu_{n}=N_{s} F(\eta) \mu_{1}, \quad<p_{T}^{2}>=\frac{<p_{T}^{2}>_{1}}{F(\eta)}
$$

At high density, $F(\eta) \simeq A^{-1 / 3}$ and $\mu_{n} \simeq N_{A}$, i. e. it is obtained the saturation of the multiplicity per participant. If $N_{s} \simeq s^{2 \lambda}, \mu_{n} \simeq s^{\lambda}$, i.e. grows with the energy slower than $N_{s}$. Outside the midrapidity, $N_{s}$ is proportional to $N_{A}$ instead of $N_{A}^{4 / 3}$. Therefore there is an additional suppression factor $N_{A}^{1 / 3}$ compared to central rapidity.

The second of equation (5) establishes the transverse size correlations which is proportional to $\frac{F(\eta)}{\left\langle p_{T}^{2}>1\right.}$ or $r_{0}^{2} F(\eta)$.

\section{String percolation and Color Glass Condensate.}

The string percolation and the glasma formed from the Color Glass Condensate have many similarities giving rise to similar predictions, as we are going to show.

One of the main scales of both models is the transverse size given by $r_{0}^{2} F(\eta)$ and $\frac{1}{Q_{s}^{2}}$ respectively which in the high density and high energy limit, both behave as $N_{A}^{-1 / 3}$ and $s^{-\lambda}$.

The effective number of independent color sources, $\langle N\rangle$ is given by the ratio between the surface occupied by strings and the transverse size

$$
<N>=\frac{\left(1-e^{-\eta}\right) R_{A}^{2}}{r_{0}^{2} F(\eta)}=\left(1-e^{-\eta}\right)^{\frac{1}{2}} \eta^{\frac{1}{2}}\left(\frac{R_{A}}{r_{0}}\right)^{2}
$$

which behaves at low density, (energy) as

$$
\eta\left(\frac{R_{A}}{r_{0}}\right)^{2} \text {, i.e. } N_{A}^{\frac{4}{3}}, \sqrt{s}^{2 \lambda}
$$

and at high density, (energy)

$$
\sqrt{\eta}\left(\frac{R_{A}}{r_{0}}\right)^{2}, \text { i. e. } N_{A}, \sqrt{s}^{\lambda}
$$

In the Glasma we have $k=Q_{s}^{2} R_{A}^{2}$ color flux tubes each producing $\frac{1}{\alpha_{s}}$ gluons in such a way that the number of color flux tubes $\mathrm{k}$, behaves as $N_{A}, s^{\lambda}$ in agreement with 
(8) [15] 16]. The origin of this behaviour in CGC, that gives rise to saturation is the non-linear behaviour of the strong color fields at low $\mathrm{x}$ produced by the color sources (gluons) located at high $\mathrm{x}$, in percolation, this behaviour is also due to the non-linear superposition of the strength of the color fields, which gives rise to a factor $n^{1 / 2}$ instead of $\mathrm{n}$ in the equation (2).

The role of the running coupling constant $\frac{1}{\alpha_{s}}$ of CGC is played in percolation of strings by the factor $\sqrt{1-e^{-\eta}}$, as it can been seen from the comparison of multiplicities in both models, i. e. $\frac{1}{\alpha_{s}} Q_{s}^{2} R_{A}^{2}$ and equation (5). As the density, or energy, increases both $\frac{1}{\alpha_{s}}$ and $\left(1-e^{-\eta}\right)^{1 / 2}$ also increase. The exponent $\lambda$ in formula (7) and (8) has been determined, using energy momentum conservation, obtaining $\lambda=\frac{2}{7}[16]$ value close to the one obtained in [17], using CGC.

The rapidity extension of the color flux tubes of the glasma are $\frac{1}{\alpha_{s}}$ which grows at high density as $\ln \left(N_{A}\right), \ln (s)$. In percolation, as the energy momentum of the clusters is the sum of the individual energy momentum of the strings, the rapidity extension of a cluster formed by $N_{s}$ strings is $\Delta y_{N}=\Delta y_{1}+2 \ln \left(N_{s}\right)$, which grows also as $\ln \left(N_{A}\right)$, $\ln (s)$. Notice, that due to that both $\mathrm{CGC}$ and percolation predict a very long rapidity correlation al LHC, b will be flat up to $\Delta \eta \geq 8$.

The inverse of the normalized fluctuations as the number of effective color sources

$$
\bar{K} \equiv \frac{<N>^{2}}{<N^{2}>-<N>^{2}}
$$

is infinity at low and high density, having a minimum at one intermediate value of $\eta$. In fact

$$
\bar{K} \equiv \frac{<N>}{\left(1-e^{-\eta}\right)^{3 / 2}}
$$

that behaves at low density as $\eta^{-\frac{1}{2}}\left(\frac{R_{A}}{r_{0}}\right)^{2}$ and at high density as $\eta^{\frac{1}{2}}\left(\frac{R_{A}}{r_{0}}\right)^{2}$ that grows like $N_{A}$ and $s^{\lambda}$. The number $\mathrm{k}$ of color flux tubes of the glasma we have seen already that has similar behavior.

In both models, the multiplicity distributions are described by negative binomial distributions [18] [19]. This distributions are determined by two parameters: the mean multiplicity and the inverse of the normalized fluctuations of the multiplicities, i. e $<n>^{2} /\left(<n^{2}>-<n>^{2}\right)$. In the glasma this parameter is $\bar{k}=<N>k_{0}$, where $k_{0}$ is the corresponding parameter of the negative binomial distribution for one string, At low density, $\bar{k} \rightarrow \infty$. The value $k_{0}=\infty$ means that for one string the negative binomial is a Bose-Einstein distribution. This result coincides with what is found in the Glasma.

Therefore $\mathrm{k}$, at low density decreases with density (energy) but as for the density increases, $\mathrm{k}$ increases with the density ( energy). This behavior means than the width of the distribution $\langle n\rangle P_{n}$ as a function of $\frac{n}{<n\rangle}$ should start to become narrower from one determined density (energy). In AA collisions at RHIC, we are in the high density sector and in fact $\mathrm{k}$ increases with the centrality.

In pp collisions, in the framework of string percolation, we expect a change on the behavior of $\mathrm{k}$ with the energy as far as we go from low density to high density. The scale can be established from the AA case as follows.

$$
\eta_{A A}=N_{s} \frac{r_{0}^{2}}{R_{A}^{2}}=\frac{N_{A}^{\frac{4}{3}}}{N_{A}^{\frac{2}{3}}}\left(\frac{r_{0}}{R_{p}}\right)^{2} N_{p}^{s}
$$


where $N_{s}$ and $N_{p}^{s}$ are the number of strings exchange in AA and pp collisions respectively. Therefore

$$
\eta_{A A}^{s}=N_{A}^{\frac{2}{3}} \eta_{p p}^{s}
$$

assuming that the critical value $\eta_{c}=1.2-1.5$ is reached in $\mathrm{Pb}-\mathrm{Pb}$ central collisions at an energy between SPS and RHIC, and taking into account the $s^{2 \lambda}$ dependence of $N_{p}^{s}$, we obtain that in pp the critical value is reached for an energy in between $6-14 \mathrm{TeV}$. Experimentally, in pp collisions $\mathrm{k}$ is decreasing from SPS up to $2.36 \mathrm{TeV}$. We expect a change on this behavior at an energy close to $7 \mathrm{TeV}[20]$.

The forward-backward correlation b, that can be written as

$$
b=\frac{1}{1+\frac{k}{<n>_{F}}}
$$

In percolation 21] 222] 23] from eqs. (10) and (5) we obtain

$$
b=\frac{1}{1+\frac{d}{\left(1-e^{-\eta}\right)^{3 / 2}}}
$$

which at low density vanishes, and at high density grows becoming $\frac{1}{1+d}$ being $\mathrm{d}$ a constant independent on the density and energy.

In $\mathrm{CGC} b$ is given by [6] []

$$
b=\frac{1}{1+\alpha_{s}^{2} c}
$$

that also grows with density and/or energy.

The strength and length of $\mathrm{b}$ are related to the high and length of the ridge structure of near side correlations. The percolation and the CGC prediction of long range correlations with length larger than eight units of rapidity means that the ridge structure will be extremely long at LHC energies.

Finally, let us mention that from the transverse size correlations and the number of effective color sources we deduce the shear viscosity which is given by $\left\langle p_{T}\right\rangle / n S_{1}$ [24] where $\mathrm{n}$ is the number of effective color sources per volume, i. e.

$$
n=\frac{<N>}{\pi R_{A}^{2} L}
$$

where $\mathrm{L}$ is the longitudinal extension, $L \simeq 1 \mathrm{fm}$. From (3) and (6) we obtain that the shear viscosity is

$$
s . v=<p_{T}>_{1} L \eta^{\frac{1}{4}} \frac{1}{\left(1-e^{-\eta}\right)^{\frac{5}{4}}}
$$

which behaves like $\eta^{-1}$ at low $\eta$ and grows like $\eta^{\frac{1}{4}}$ at high density, having a minimum close to the critical value of percolation.

Recently, in string percolation has been computed the equation of state. The results for the energy density, speed sound, energy density and pressure [25] are just on the same curve computed in lattice QCD.

All the similarities seen on the behavior of the main observables with the density and energy in string percolation and CGC, including the prediction of very long range rapidity correlations, indicate that both models are complementary views of the same physical picture. 


\section{Acknowledgements}

Most of the work reported here was done in collaboration with J. Dias de Deus. I thank also J. G. Milhano and I. Bautista for helping me in this work. I thank the support of the project FPA2008-01177 of Spain, and of the Xunta de Galicia.

\section{References}

[1] B. I. Abelev et al. [STAR Collaboration], Phys. Rev. Lett. 103 (2009) 172301, T. Tarnowsky PhD Thesis nucl-exp 08071941

[2] B. B. Back et al. [PHOBOS Collaboration], Phys. Rev. C 74 (2006) 011901

[3] S. S. Adler et al. [PHENIX Collaboration], Phys. Rev. C 76, 034903 (2007)

4 A. Capella, U. Sukhatme, C. I. Tan and J. Tran Thanh Van, Phys. Rept. 236, 225 (1994)

[5] N. S. Amelin, N. Armesto, C. Pajares and D. Sousa, Eur. Phys. J. C 22, 149 (2001)

[6] N. Armesto, L. McLerran and C. Pajares, Nucl. Phys. A 781, 201 (2007) N. Armesto, M. A. Braun and C. Pajares, Phys. Rev. C 75, 054902 (2007)

[7] Y. V. Kovchegov, E. Levin and L. D. McLerran, Phys. Rev. C 63, 024903 (2001)

[8] K. Dusling, F. Gelis, T. Lappi and R. Venugopalan, Nucl. Phys. A 836, 159 (2010)

[9] Y. L. Yan, D. M. Zhou, B. G. Dong, X. M. Li, H. L. Ma and B. H. Sa, Phys. Rev. C 79, 054902 (2009).

[10] A. Bzdak and K. Wozniak, Phys. Rev. C 81, 034908 (2010) A. Bzdak, Phys. Rev. C 80, 024906 (2009)

[11] N. Armesto, M. A. Braun, E. G. Ferreiro and C. Pajares, Phys. Rev. Lett. 77, 3736 (1996)

[12] M. A. Braun and C. Pajares, Eur. Phys. J. C 16, 349 (2000) M. A. Braun and C. Pajares, Phys. Rev. Lett. 85, 4864 (2000)

[13] L. D. McLerran and R. Venugopalan, Phys. Rev. D 49, 2233 (1994)

[14] T. Lappi and L. McLerran, Nucl. Phys. A 772, 200 (2006)

[15] D. Kharzeev, E. Levin and M. Nardi, Nucl. Phys. A 747 (2005) 609

[16] J. Dias de Deus, M. C. Espirito Santo, M. Pimenta and C. Pajares, Phys. Rev. Lett. 96 (2006) 162001

[17] J. L. Albacete, N. Armesto, J. G. Milhano, C. A. Salgado and U. A. Wiedemann, Phys. Rev. D 71, 014003 (2005) N. Armesto, C. A. Salgado and U. A. Wiedemann, Phys. Rev. Lett. 94, 022002 (2005)

[18] F. Gelis, T. Lappi and L. McLerran, Nucl. Phys. A 828, 149 (2009)

[19] J. Dias de Deus, E. G. Ferreiro, C. Pajares and R. Ugoccioni, Eur. Phys. J. C 40, 229 (2005)

[20] J. Dias de Deus, E. G. Ferreiro, C. Pajares and R. Ugoccioni, Phys. Lett. B 601, 125 (2004)

[21] P. Brogueira, J. Dias de Deus and C. Pajares, Phys. Lett. B 675, 308 (2009)

[22] P. Brogueira and J. Dias de Deus, Phys. Lett. B 653, 202 (2007)

[23] N. S. Amelin, N. Armesto, M. A. Braun, E. G. Ferreiro and C. Pajares, Phys. Rev. Lett. 73, 2813 (1994).

[24] J. Dias de Deus and C. Pajares, Phys. Lett. B 642, 455 (2006)

[25] R. P. Scharenberg, B .K. Srivastava, A. S. Hirsch, to appear. 